Tohoku J. exp. Med., 1980, 132, 243-244

Short Report

\title{
A Prototype Apparatus for Microexplosion Cystolithotripsy
}

\author{
Hiroki Watanabe, Kousuke Watanabe, Kazuo Shino* \\ and Senzo Oinuma* \\ Department of Urology, Kyoto Prefectural University of \\ Medicine, Kyoto 602 and *National Chemical Laboratory, \\ MITI, Tsukuba 305
}

Watanabe, H., Watanabe, K., Shirno, K. and Oinuma, S. A Prototype Apparatus for Microexplosion Cystolithotripsy. Tohoku J. exp. Med., 1980, 132 (2), 243-244 - We developed a prototype apparatus for microexplosion lithotripsy in the urinary bladder according to our original idea. A model calculus in a pig bladder was successfully crushed by the apparatus and was removed by aspiration. - microexplosion; urinary calculi; lithotripsy; cystoscope; urinary bladder

We first put forward the idea of microexplosion lithotripsy in 1975. Our first report on the fundamental experiments concerning this technique was published in 1977 (Watanabe and Oinuma 1977). Since then, a systematic research project has been performed on various subjects necessary to the technique (Murata et al. 1977, 1978; Kaneko et al. 1979; Oki et al. 1980). On the basis of these data, we recently developed a prototype apparatus for microexplosion lithotripsy in the urinary bladder.

\section{Apparatus}

Five $\mathrm{mg}$ of lead azide explosives were loaded in one end of a special cylindrical explosive chamber $3 \mathrm{~mm}$ in diameter and $20 \mathrm{~mm}$ in length and made of stainless steel. This end of the chamber was coated with epoxy resin to make it water tight. A platinum

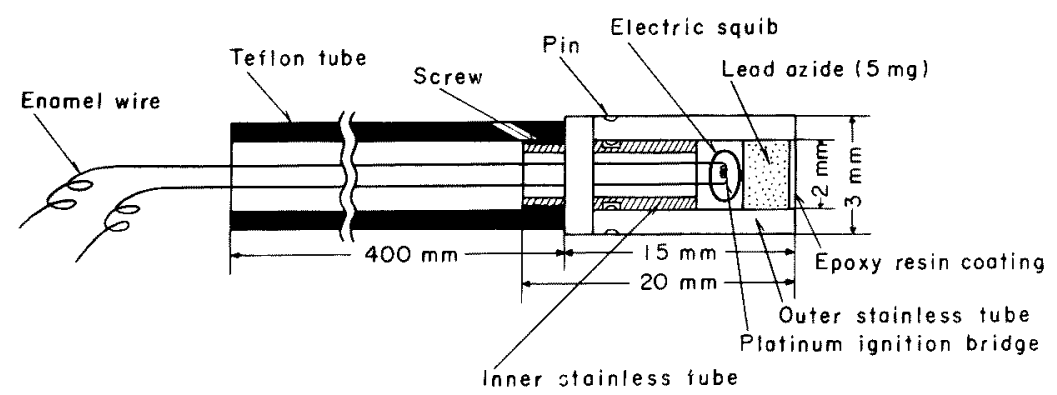

Fig. 1. The explosive chamber with $5 \mathrm{mg}$ of lead azide and an ignition bridge inside it. A flexible teflon tube is attached so that the whole system functions as an "explosive catheter".

Received for publication, May 19, 1980.

This work was supported by grants from the Ministry of Education, Science and Culture and from the Science and Technics Agency, Japan. 
ignition bridge was set in the other end of the chamber. The outside of the chamber on this end was shaped like a screw and a flexible teflon tube, approximately $400 \mathrm{~mm}$ in length, of the same diameter as the chamber, was attached over the screw. Lead wires from the bridge were guided through the inside of the teflon tube. Thus the whole system functioned as an "explosive catheter" (Fig. 1).

An ordinary Bürger-type surgical cystoscope was somewhat modified to allow the insertion of the "explosive catheter". By operating the deflector of the cystoscope, the chamber at the tip of the "catheter" could be moved to any position required in the bladder under direct observation (Fig. 2).

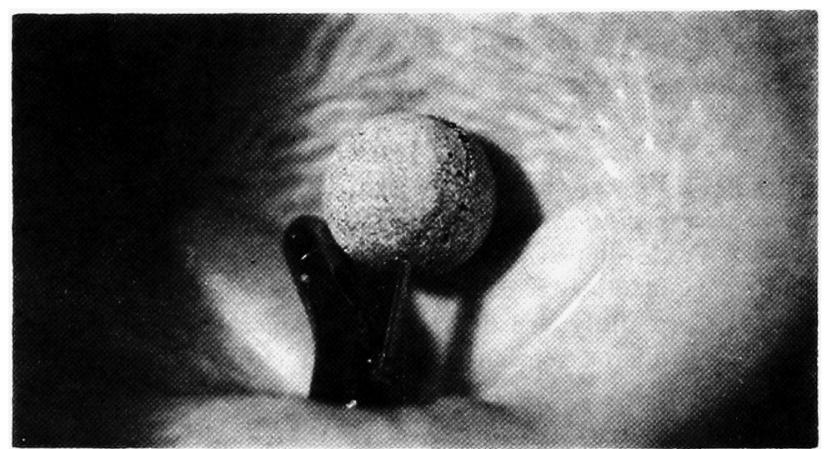

Fig. 2. The explosive chamber is brought into contact with a model calculus through a modified Bürger's cystoscope.

\section{Results}

Two female pigs about $60 \mathrm{~kg}$ in weight were prepared to test the apparatus. The urinary bladder was incised under general anesthesia and a specially developed spherical model calculus, $20 \mathrm{~mm}$ in diameter, made of a mixture of calcium sulfate and grained sand, of which the compressive and tensile strength approximated those of the human urinary calculi, was put into the bladder. The incision was closed by suture. The bladder was filled with water. The calculus was easily located and the chamber was brought into contact with it. A $3 \mathrm{~V}$ A.C. electric source was connected to the lead wires. The explosives were detonated and the calculus was crushed. In one case two explosions were needed to crush the calculus into fragments, while in the other case one shot was sufficient. The crushed fragments of the calculus were completely aspirated from the urethra with a Thompson's aspirator. Neither injury nor bleeding was observed in the bladder wall after the procedure.

Thus the test was completed successfully. Further evaluation on safety factors over a definite period of time will, however, be necessary until the technique can be applied to the human body.

\section{References}

1) Kaneko, H., Watanabe, H., Takahashi, T., Watanabe,K., Akiyama, K., Kondo, K., Furue, H. \& Oinuma, S. (1979) Strength of wet and dry urinary calculi. Jap. J. Urol., 70, 61-66.

2) Murata, S., Watanabe, H., Takahashi, T., Watanabe, K., Furue, H. \& Oinuma, S. (1977) Construction and strength of urinary calculi. Jap. J. Urol., 68, 249-257.

3) Murata, S., Watanabe, H., Watanabe, K., Takahashi, T., Akiyama, K., Oinuma, S. \& Yoshikawa, M. (1978) Amino acids in urinary calculi. Jap. J. Urol., 69, 33-39.

4) Oki, F., Saito, T. \& Watanabe, H. (1980) A fluid dynamical theory of microexplosion for medicine and biology. Tohoku.J. exp. Med., 133, in press.

5) Watanabe, H. \& Oinuma, S. (1977) Development of special explosive for the experiments. Jap. J. Urol., 68, 243-248. 